\title{
Review
}

\section{The fly caspases}

\author{
S Kumar, ${ }^{\star, 1}$ and J Doumanis ${ }^{1}$ \\ ${ }^{1}$ Hanson Centre for Cancer Research, Institute of Medical and Veterinary \\ Science, PO Box 14, Rundle Mall, Adelaide, SA 5000, Australia \\ * Corresponding author: S Kumar, Hanson Centre for Cancer Research, Institute \\ of Medical and Veterinary Science, PO Box 14, Rundle Mall, Adelaide, SA 5000, \\ Australia; Tel: +61-8-8222-3738; Fax: +61-8-8222-3139; \\ E-mail: sharad.kumar@imvs.sa.gov.au
}

Received 14.6.00; revised 26.7.00; accepted 27.7.00

Edited by G Melino

\begin{abstract}
Caspases, a group of cysteine proteases, constitute the effector arm of the cell death machinery. There are seven caspases known in Drosophila, three of which contain long amino-terminal prodomains. Although, compared to mammalian caspases, much less is known about the biology of Drosophila caspases, many studies have shown that caspases are essential for programmed cell death in the fly and are likely to be regulated in ways similar to their mammalian counterparts. Studies on fly caspases have revealed some new insights on cell death regulation. For example, the transcript for the fly caspase DRONC is regulated by the hormone ecdysone during programmed cell death in specific tissues. Recent data on DRONC also suggest that some fly caspases may have unique substrate specificities not ascribed to mammalian caspases. The presence of multiple caspases in Drosophila indicates that apoptotic pathways in insects are likely to be as complex as in vertebrates. Cell Death and Differentiation (2000) 7, 1039-1044.
\end{abstract}

Keywords: DRONC; DCP-1; DRICE; DECAY; DREDD; DAMM; STRICA

\section{Introduction}

Caspases are cysteine proteases related to the prototypic member of the family, the Caenorhabditis elegans death effector CED-3..$^{1-4}$ These proteases, which cleave their substrates following an aspartate residue, are normally present as inactive precursors in cells. Upon receiving an apoptotic signal, the pro-forms (zymogens) of caspases undergo proteolytic processing to generate active enzyme. $^{1-4}$ In mammals, there are at least 14 caspases discovered so far, some of which are involved in apoptosis, whereas others are believed to be primarily responsible for the activation and regulation of proinflammatory cytokines. ${ }^{1-4}$ The first insect caspase, Sf caspase-1, was cloned from Spodoptera frugiperda. ${ }^{5}$ There are a total of seven caspases in Drosophila melanogaster, five of which, named DCP-1, DREDD/DCP-2, DRICE, DRONC and DECAY have been described in the published literature. ${ }^{6-11}$ The work on other two caspases, DAMM (GenBank accession no. AF240763) and STRICA/DREAM (GenBank accession nos. AF242735 and AF275814) has not yet been published. The genome sequence of $D$. melanogaster predicts a total of eight caspases in the fly, ${ }^{12}$ however, after repeated homology searches we have only found sequence encoding seven caspases in the fly. This article summarises what is currently known about the biology of caspases in Drosophila.

\section{Structure and expression of fly caspases}

Activated caspases are tetrameric structures consisting of two large and two small subunits. ${ }^{1-4}$ Each heterologous pair of subunits is derived from a single caspase precursor (procaspase) molecule. Following apoptotic signalling, procaspases undergo cleavage and activation. Two types of caspases have been found in all species: class I caspases containing long prodomains amino terminal to the caspase domain that is comprised of the two subunits, and class II caspases which lack a prodomain or have a very short prodomain. ${ }^{1-4,13,14}$ The long prodomains of class I caspases contain specific protein-protein interaction motifs, such as the caspase recruitment domain (CARD) or death effector domain (DED).$^{15}$ In mammals, the caspase-9 CARD is required for the recruitment of procaspase- 9 by Apaf- 1 via a CARD present at the amino terminus of Apaf-1, whereas one of the two DEDs in caspase-8 interacts with the DED in the adaptor molecule FADD. ${ }^{13-17}$ In both cases, recruitment of procaspase molecules via their respective adaptors is necessary for promoting their autocatalytic activation. Similar to mammalian caspases, Drosophila caspases can also be divided into long (class I) and short (class II) prodomain caspases. Among the seven caspases, DREDD, DRONC and STRICA contain long prodomains (Figure 1). DREDD contains two DEDs in its prodomain region whereas DRONC is the only CARD containing caspase in flies. ${ }^{8-10}$ STRICA, the third fly caspase with a long amino-terminal region, contains a Ser/ Thr-rich prodomain that lacks any CARD or DED like structures (Doumanis and Kumar, unpublished results). The significance and function of this unusual prodomain in STRICA is not currently known, and similar prodomains have not been reported in mammals. The other four fly caspases, DCP-1, DRICE, DECAY and DAMM, lack long prodomains and are thus similar to class II caspases in mammals. ${ }^{13}$

A comparison of the amino acid sequences of fly and mammalian caspases reveals the evolutionary relationship between various caspases. In Drosophila, DCP-1 and DRICE are highly homologous to each other, and Sf caspase-1 is likely to be the Spodoptera homologue of 


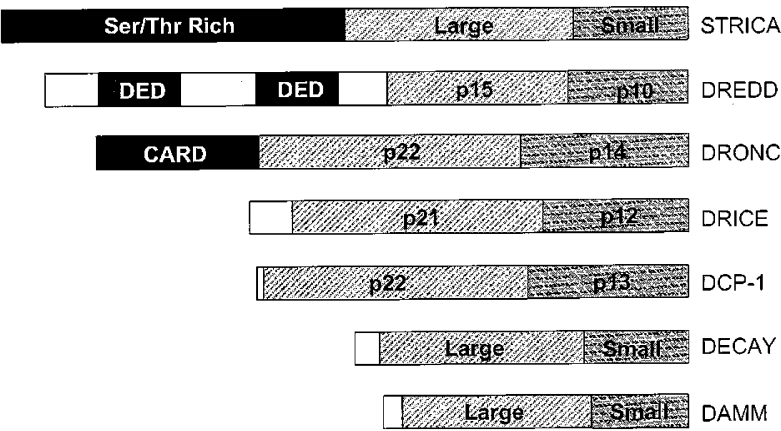

Figure 1 Primary structure of various Drosophila caspases. The prodomains of class I caspases DRONC and DREDD contain protein-protein interaction motifs, CARD and DEDs, respectively. However, the Ser/Thr-rich prodomain of STRICA bears no significant homology to any other prodomain. Where known, the sizes of the large and small subunits are shown. Subunit boundaries for STRICA, DECAY and DAMM, for which there is no such information currently available, are arbitrary

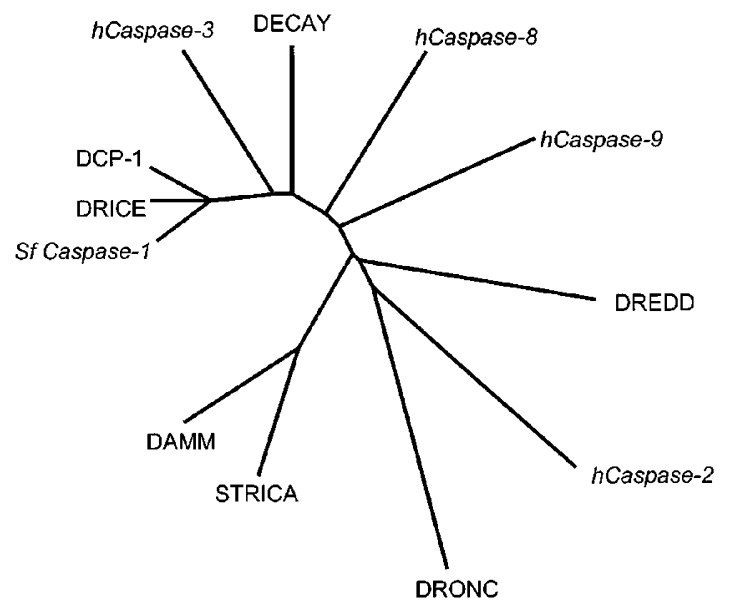

Figure 2 A diagram illustrating the phylogenetic relationship between some of the proapoptotic human (h) caspases, Spodoptera frugiperda (Sf) caspase1 and the Drosophila caspase family members. Non-Drosophila caspases are shown in italics. Class II caspases, DRICE, DCP-1 and DECAY are most highly related to mammalian Caspase-3. DAMM and STRICA, while being highly similar to each other, form a group distinct from other Drosophila caspases. The class I caspases, DREDD and DRONC are more closely related to human caspase-2 than to the human caspases -8 and -9

DRICE (Figure 2). DECAY is the next closest caspase to DRICE and DCP-1. All these three class II caspases share high degree of homology with the mammalian caspase-3. Despite the presence of two DEDs in its prodomain, DREDD shows more overall similarity to the CARD containing caspase-2, than to the DED containing caspase-8. DRONC is also most similar to caspase-2, rather than its predicted functional homologue caspase-9. DAMM and STRICA are distantly related to other fly caspases. Although DAMM and STRICA differ in the length of their prodomains, these two caspases share highly related caspase domains (Figure 2).

The transcripts for $d c p-1$, drice, dredd, dronc and decay are present at a relatively high level in preblastoderm embryos prior to the onset of zygotic transcription. ${ }^{6-8,10,11}$
As cell death in embryos does not start until much later, ${ }^{18}$ the role of this maternally deposited caspase RNA is not understood. In later embryonic stages dcp-1, dronc and decay transcripts are uniformly distributed in the embryo, but become more restricted towards the end of embryogenesis. $6,10,11$ Interestingly, in late third instar larvae, dronc mRNA is dramatically up-regulated in salivary glands and midgut before histolysis of these tissues. ${ }^{10}$ Exposure of salivary glands and midgut isolated from second instar larvae to ecdysone results in a massive increase in dronc mRNA levels suggesting that DRONC is an effector of steroid-mediated apoptosis during insect metamorphosis. ${ }^{10}$ Among the published Drosophila caspases, dredd shows the most dramatic expression pattern. While in early embryos dredd transcript is uniformly distributed, from stage 11 of embryogenesis, it becomes highly concentrated in cells specified to die. ${ }^{8}$ During oogenesis $d c p-1$, dredd, dronc and decay mRNA is detected in egg chambers at various stages. ${ }^{6,8,10,11}$ Relatively high level of decay ${ }^{11}$ and damm expression (Harvey and Kumar, unpublished data) is seen in adult flies. The overlapping patterns of expression of many fly caspases suggest that several caspases may be necessary for programmed cell death in a given pathway.

\section{Functions of fly caspases}

Of all the fly caspases, at present specific mutations are only available for $d c p-1$. $d c p-1$ null mutations are larval lethal but do not show any abnormality in embryonic cell death. ${ }^{6}$ Since $d c p-1$ mRNA is likely to be maternally deposited, it is possible that the maternal protein contributes to embryonic cell deaths. ${ }^{6}$ The $d c p-1$ null larvae that survive to third instar display normal central nervous system but lack gonads and imaginal discs and have fragile trachea. ${ }^{6}$ In addition, these larvae have melanotic tumours. ${ }^{6}$ Female flies carrying the $d c p-1^{-}$germ line clones are sterile due to a defect in the transfer of nurse cell cytoplasmic contents to developing oocytes. ${ }^{19}$ Nurse cells lacking $d c p-1$ function show abnormal cytoskeletal reorganisation and nuclear breakdown that occurs during the cytoplasmic dumping process. ${ }^{19}$ Nurse cell death has been described in detail in an accompanying article. Transgenic expression of truncated DCP-1 that lacks the amino-terminal 28 residues, but not the full length DCP-1, using an eye specific promoter, results in a small and rough eye phenotype. ${ }^{20}$ In transgenic expression models, reaper (rpr) and grim, but not hid enhanced the eye phenotype of truncated $d c p-1$ flies suggesting that RPR and GRIM, but not $\mathrm{HID}$, can activate DCP-1 in vivo. ${ }^{20}$

As stated above, the mRNA for dredd accumulates in preapoptotic cells. Interestingly, such accumulation of dredd message is not seen in the H99 mutant that is deficient in rpr, hid and grim, whereas ectopic expression of rpr, hid or grim promotes dredd mRNA accumulation. ${ }^{8}$ These results suggest that dredd transcript indeed accumulates in cells already specified to undergo apoptosis. ${ }^{8}$ Consistent with these observations, signalling by RPR, HID and GRIM results in processing of DREDD to its active form in transfected Drosophila S2 cells. ${ }^{8}$ Further evidence for a function of DREDD in apoptosis comes from 
the observation that heterozygosity at the dredd locus suppresses cell death induced by the ectopic expression of rpr, grim and hid in transgenic models, indicating that the concentration of DREDD can be a rate limiting step in apoptosis mediated via the death activators rpr, grim and hid. $^{8}$ In addition to its function in cell death, DREDD also plays a key role in the innate immune response ${ }^{21}$ by regulating the activation of $\mathrm{RELISH}$, a member of the $\mathrm{NF} \kappa \mathrm{B}$ family (Abrams and Lemaitre, personal communication). Flies mutated at the dredd locus fail to induce the synthesis of anti-microbial peptides and are highly susceptible to infection by gram negative bacteria. ${ }^{21}$

Ectopic overexpression of DRICE sensitises Drosophila S2 cells to apoptotic stimuli and expression of an aminoterminally truncated form of DRICE lacking the first 80 amino acid residues, rapidly induces apoptosis in Drosophila cells. ${ }^{7,22}$ However, when expressed in Drosophila eye, neither the full length nor DRICE lacking 28 amino terminal residues, shows any overt effects. ${ }^{20}$ Induction of apoptosis by rpr overexpression or by cycloheximide or etoposide treatment of Drosophila cells results in proteolytic processing of DRICE. ${ }^{7}$ Using antibodies specific to DRICE, it was found that immunodepletion of DRICE from Drosophila S2 cell lysates is sufficient to remove most measurable in vitro apoptotic activity, and that addition of recombinant DRICE to such immunodepleted lysates restores apoptotic activity. ${ }^{22}$ These results point to an essential role for DRICE in at least S2 cells.

Ectopic expression of DRONC induces cell death in Schizosaccharomyces pombe, Drosophila and mammalian cells, and in developing Drosophila tissues. ${ }^{10,23,24}$ The heterozygosity at the dronc locus or the expression of a catalytically inactive DRONC mutant suppress the eye phenotype caused by rpr, and hid, consistent with the idea that DRONC functions in the RPR and HID pathway. ${ }^{23,24}$ DRONC also interacts, both biochemically and genetically, with the CED-4/Apaf-1 fly homologue, DARK (Quinn et al, unpublished data; ${ }^{25}$ ). Furthermore, loss of DRONC function in early Drosophila embryos using the RNA interference technique results in a decrease in apoptosis, indicating that DRONC is required for programmed cell death during embryogenesis (Quinn et al, unpublished data). These results suggest that DRONC is a key caspase in mediating developmentally programmed cell death in Drosophila.

Ectopic expression of DECAY has a weak apoptotic effect in mammalian cells, ${ }^{11}$ but its function in Drosophila cell death pathways is completely obscure at present. Similarly, the functions of DAMM and STRICA, both of which can induce cell death in vitro to some extent (Harvey et al, unpublished data), are not fully understood.

\section{Activators and inhibitors of fly caspases}

In $C$. elegans the activation of CED-3 requires interaction with CED-4. ${ }^{26}$ In an analogous scenario, mammalian caspase-9 requires a CED-4-like protein Apaf-1 for its activation. ${ }^{13-17} \mathrm{~A}$ single CED-4/Apaf-1 homologue, variously called DARK/ DAPAF-1/HAC-1, has been described in Drosophila. ${ }^{25,27,28}$ Like most of the caspases, the transcript for dark/dapaf-1/hac1 appears to be maternally deposited in the zygote, but in later stages of development, hac-1 mRNA is expressed in a regulated manner in regions of tissues that contain cells undergoing apoptosis. ${ }^{28}$ Interestingly, hac-1 transcription is upregulated by UV and X-ray irradiation. ${ }^{28}$ Loss-of-function mutations in dark/dapaf-1/hac-1 suppress developmentally programmed cell death, resulting in hyperplasia of the central nervous system, and other abnormalities including ectopic melanotic tumours and defective wings. ${ }^{25,27,28}$ Although requirement of DARK/DAPAF-1/HAC-1 in the activation of specific caspases has not been demonstrated as yet, a significant amount of data suggest that this molecule plays a key function upstream of caspases. DARK/DAPAF-1/HAC-1 can mediate caspase activation in vitro. ${ }^{25,27,28}$ Furthermore, its function is inhibited by caspase inhibitors and the molecule associates with DRONC and DREDD, two of the predicted apical caspases. ${ }^{25,27,28}$ A dominant negative version of DREDD suppresses DARK-induced cell death. ${ }^{25}$ In vivo, heterozygosity for dark/hac-1 results in suppression of the eye ablation phenotype caused either by the overexpression of $d c p-1^{28}$ or dronc (Quinn et al, unpublished data). The function of DARK/DAPAF-1/HAC-1 in RPR, HID and GRIM mediated cell death is somewhat contentious at present. Rodriguez et al reported that in dark mutants, cell death induced by the ectopic expression of rpr, hid and grim is substantially reduced. ${ }^{27}$ Kanuka et al found that while rprinduced cell death in the eye was substantially inhibited in dapaf-1 mutants, hid-induced apoptosis was not affected. ${ }^{25}$ On the contrary, Zhou et al failed to see any significant effect on killing by rpr, hid or grim in hac-1 mutants. ${ }^{28}$ It is noteworthy that Kanuka et al and Zhou et al used a relatively weak allele of dapaf-1/hac-1 in their experiments which might, in part, explain their negative results. Nevertheless, further experiments are necessary to delineate whether DARK/DAPAF-1/ HAC-1 plays a role in the RPR, HID and GRIM mediated apoptotic pathways.

As discussed in detail elsewhere in this issue, members of the IAP family of proteins can inhibit caspase function. In Drosophila, DIAP1 appears to be a key regulator of several caspases, including DCP-1, DRICE and DRONC. ${ }^{23,29-32}$ Genetic and biochemical studies indicate that DIAP1 function is required for cell survival by blocking the activation of caspases, and that RPR, HID and GRIM promote apoptosis by disrupting the DIAP1-caspase interaction. $^{31,32}$ Coexpression of DIAP1 in the developing Drosophila eye rescues the eye ablation phenotype induced by DRONC expression, whereas heterozygosity at the diap1 locus enhances the DRONC eye phenotype, consistent with a role for endogenous DIAP-1 in suppression of DRONC activation. ${ }^{23,24}$

\section{Role of cytochrome $c$ in caspase activation in Drosophila}

In mammals, the activation of caspase- 9 requires cytochrome $c,{ }^{33}$ and in mice in which the cytochrome $c$ gene has been deleted by homologous recombination, the caspase-9/Apaf-1 pathway is severely impaired. ${ }^{34}$ Cytochrome $c$ released from the mitochondria binds to Apaf-1 and enables the oligomerisation of Apaf-1 molecules. ${ }^{13-17}$ Oligomerised Apaf-1 can then recruit procaspase- 9 and thereby promote its proximity- 
induced autocatalytic activation. ${ }^{13-17}$ Unlike in mammals, CED-4-mediated CED-3 activation in $C$. elegans does not require cytochrome $c$, suggesting that cytochrome $c$ function in apoptosis evolved late in more complex organisms. The role of cytochrome $c$ in Drosophila caspase activation is not fully understood. Although structurally DARK/DAPAF-1/HAC1, the Drosophila CED-4 homologue, is more similar to its mammalian counterpart Apaf-1 than to CED-4, in that it consists of several WD40 repeats not found in CED- $4,25,27,28$ it is as yet unclear whether it requires cytochrome $c$ for its oligomerisation. Kanuka et al have shown that addition of cytochrome $c$ and dATP to Drosophila embryo extracts results in a twofold enhancement of DEVDase activity, that was not seen in extracts prepared from dapaf-1 mutant embryos. ${ }^{25}$ The cytochrome c/dATP induced caspase activity was also inhibited by an ATPase inhibitor suggesting that the ATPase activity of DAPAF-1 may be necessary for its ability to mediate caspase activation. ${ }^{25}$

There are conflicting reports of cytochrome $c$ release from the mitochondria of Drosophila cells during apoptosis. Kanuka et al found that in S2 cells, overexpression of rpr or treatment with staurosporine or cycloheximide resulted in the release of cytochrome $c$ into the cytosol. ${ }^{25}$ On the other hand, Varker et al demonstrated an alteration in cytochrome $c$ conformation, as evident by display of an otherwise hidden epitope, in Drosophila tissues, preceding apoptosis. $^{35}$ This alteration occurs without release of the protein into the cytosol. Surprisingly, caspase activity is necessary and sufficient to mediate changes in cytochrome $c$, suggesting that involvement of cytochrome $c$ was downstream of caspase activation. ${ }^{35}$ In cell-free studies, caspase activation was triggered by mitochondria from apoptotic cells but not from those from healthy cells. ${ }^{35}$ These observations suggest that in the fly the function of cytochrome $c$ in caspase activation may be somewhat different from its role in mammalian cells. If cytochrome $c$ is not released from mitochondria, it is unclear how it activates cytoplasmic caspases. Although DARK/DAPAF-1 has been shown to bind cytochrome $c$ in coimmunoprecipitation experiments, ${ }^{25,27}$ it is not entirely clear whether DARK/DAPAF-1 can directly interact with cytochrome $c$ in vivo, and if it does, where in the cell this occurs. Both from the mechanistic and the evolutionary point of view, these are important questions to address.

\section{Substrate specificity and downstream targets of fly caspases}

Mammalian caspases have been divided into three groups based on their substrate specificities. ${ }^{4}$ Group 1 caspases (caspase-1, -4, -5 and -13) prefer a bulky hydrophobic residue in $\mathrm{P}_{4}$, group 2 caspases (caspase-2, -3 and -7 ) have a strict requirement for an $A s p$ in the $P_{4}$ position, whereas group 3 caspases (caspase-6, -8, -9 and -10 ) prefer a branched chain aliphatic amino acid in $\mathrm{P}_{4}$. Group 2 caspases, particularly caspase-3, with a $P_{4}-P_{1}$ specificity of DxxD are considered to be activated by upstream caspases such as caspase- 8 and caspase-9, and mediate the cleavage of cellular proteins during apoptosis. ${ }^{4}$ By sequence comparison, DCP-1, DRICE and DECAY appear related to caspase-3, and indeed all these caspases can cleave several proteins following DxxD. In vitro DRICE, DCP-1 and DECAY can cleave poly(ADPribose) polymerase.,7,11 Furthermore, DCP-1 and DRICE also cleave baculovirus p35 and Drosophila lamin DmO. 6,7,22 One of the key substrates for caspase- 3 in mammalian cells is DNA fragmentation factor-45 (DFF45) or inhibitor of caspase activated DNase (ICAD). ${ }^{36,37}$ The cleavage of ICAD by caspase-3 is required for the release and activation of CAD, which mediates DNA fragmentation. ${ }^{37}$ In an analogous scenario, caspase-3 and DRICE have been shown to mediate cleavage of Drosophila ICAD (dICAD) recently. ${ }^{38}$ However, unlike in mammals, caspase-mediated CAD cleavage was also necessary for dCAD activation. ${ }^{38}$ The DRICE-like caspase from Spodoptera, Sf caspase-1, has been shown to cleave nuclear immunophilin FKBP46 and p35. ${ }^{5}$ These observations indicate that caspase-3-like fly caspases, DRICE in particular, may be key effectors of proteolysis during insect cell apoptosis. There are over 75 caspase substrates described in mammalian cells. Therefore there are likely to be many more substrates of fly caspases yet to be discovered.

The optimal cleavage specificities for DCP-1 and DRONC have been determined using synthetic combinatorial peptide libraries. ${ }^{20,24}$ As expected, DCP-1 substrate specificity is identical to caspase-3, with the optimal tetrapeptide substrate being DEVD. ${ }^{20}$ On the other hand, DRONC can tolerate a number of amino acids at $P_{3}$ and $\mathrm{P}_{4}$, but shows a strong preference for Thr, lle or Val at $\mathrm{P}_{2}{ }^{24}$ However, in addition to its aspase activity, DRONC can also cleave following a Glu in $\mathrm{P}_{1} \cdot{ }^{24}$ In fact DRONC self cleavage in $E$. coli occurs following a TQTE sequence in proDRONC. ${ }^{24}$ This unusual dual substrate cleavage activity has not been ascribed to any other caspases, and as the name suggests, caspases are believed to have a strict requirement for an Asp at $\mathrm{P}_{1}$ position. ${ }^{1-4}$ DRONC has a unique catalytic site not found in other known caspases (Figure 3), which may explain in part its unusual substrate preference. Interestingly, DRONC does not interact with, or cleave $\mathrm{p} 35,{ }^{23,24}$ although it is able to cleave and activate

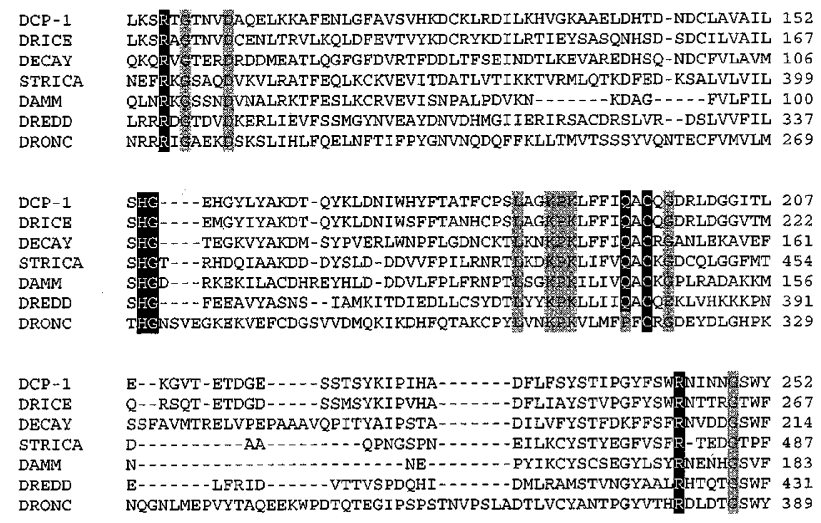

Figure 3 An alignment of the partial enzymatic domains of the Drosophila caspases. Based on published work on human caspases, ${ }^{4}$ the residues highlighted in black are predicted to contribute to the active site of the enzyme. These residues are likely to be required for the catalytic activity of the caspases and stabilisation of the $\mathrm{P}_{1}$ aspartate residue in the substrate. Other conserved residues are shown in grey 
DRICE at a TETD sequence, consistent with its predicted function as an apical caspase. ${ }^{23}$ Experiments with $\mathrm{Sf}$ caspase-1 show that the cleavage of this caspase at the TETD site, that generates the two subunits, is mediated by a p35 insensitive caspase. ${ }^{39}$ Therefore it is possible that DRONC is responsible in vivo for the activation of DRICE in Drosophila and Sf caspase-1 in Spodoptera.

\section{Concluding remarks}

The study of fly caspases is still in a relatively nascent stage, however published work so far has clearly demonstrated the essential role for caspases in programmed cell death in Drosophila. Nevertheless, there are a number of key issues that require addressing. These include: the function of fly caspases in processes other than cell death; the role of cytochrome $c$ in caspase activation; identification of cellular targets of fly caspases; functions of individual caspases in temporally and spatially controlled cell death during development and metamorphosis; identification of developmental cues that regulate caspase expression, activation and function; and specifying how the caspase cascade links to the upstream signalling molecules. Of course many of these questions also remain unanswered in the mammalian system. In particular, despite the availability of many caspase knockouts in mice, we still need to learn a great deal about caspase regulation during development. The discovery of dronc regulation by ecdysone suggests that one way of regulating cell death during development and tissue remodelling may be by transcriptionally regulating a death effector such as a caspase. Similar mechanisms regulated by hormones or retinoic acid may also exist in mammals, and will be worth investigating. Drosophila is a powerful system to study the function of individual genes and to define interactions between different molecules of a pathway. Once individual caspase mutants of Drosophila become available it should be relatively easy to study the roles and regulation of various caspases in cell death. Given the complexity of the biological pathways, there may still be many surprises to uncover. These will be keenly awaited.

\section{Acknowledgements}

We thank John Abrams and Natasha Harvey for helpful comments and discussions. The work in our laboratory is supported by the Wellcome Trust and the National Health and Medical Council of Australia. S Kumar is a Wellcome Senior Fellow in Medical Science. J Doumanis is a Dawes Postgraduate Scholar.

\section{References}

1. Kumar Sand Lavin MF (1996) The ICE family of cysteine proteases as effectors of cell death. Cell Death Differ. 3: 255-267

2. Thornberry NA and Lazebnik Y (1998) Caspases: enemies within. Science 281: $312-316$

3. Cryns V and Yuan J (1999) Proteases to die for. Genes Dev. 12: 1551 - 1570

4. Nicholson DW (1999) Caspase structure, proteolytic substrates, and function during apoptotic cell death. Cell Death Differ. 6: 1028-1042
5. Ahmad M, Srinivasula SM, Wang L, Litwack G, Fernandes-Alnemri T and Alnemri ES (1997) Spodoptera frugiperda caspase-1, a novel insect death protease that cleaves the nuclear immunophilin FKBP46, is the target of the baculovirus antiapoptotic protein p35. J. Biol. Chem. 272: 1421-1424

6. Song Z, McCall K and Steller H (1997) DCP-1, a Drosophila cell death protease essential for development. Science 275: 536-540

7. Fraser AG and Evan GI (1997) Identification of a Drosophila melanogaster ICE/ CED-3-related protease, drICE. EMBO J. 16: 2805-2813

8. Chen P, Rodriguez A, Erskine R, Thach T and Abrams JM (1998) Dredd, a novel effector of the apoptosis activators reaper, grim, and hid in Drosophila. Dev. Biol. 201: $202-216$

9. Inohara N, Koseki T, Hu Y, Chen S and Núñez G (1997) CLARP, a death effector domain containing protein interacts with caspase-8 and regulates apoptosis. Proc. Natl. Acad. Sci. USA 94: 10717-10722

10. Dorstyn L, Colussi PA, Quinn LM, Richardson Hand Kumar S (1999) DRONC, an ecdysone inducible Drosophila caspase. Proc. Natl. Acad. Sci. USA 96: 4307 4312

11. Dorstyn L, Read SH, Quinn LM, Richardson H and Kumar S (1999) DECAY, a novel Drosophila caspase related to mammalian caspase-3 and caspase-7. J. Biol. Chem. 274: 30778-30783

12. Rubin GM, Yandell MD, Wortman JR, Miklos GLG, Nelson CR, Hariharan IK, Fortini ME, Li PW, Apweiler R, Fleischmann W, Cherry JM, Henikoff S, Skupski MP, Misra S, Ashburner M, Birney E, Boguski MS, Brody T, Brokstein P, Celniker SE, Chervitz SA, Coates D, Cravchik A, Gabrielian A, Galle RF, Gelbart WM, George RA, Goldstein LSB, Gong F, Guan P, Harris NL, Hay BA, Hoskins RA, Li J, Li Z, Hynes RO, Jones SJM, Kuehl PM, Lemaitre B, Littleton JT, Morrison DK, Mungall C, O'Farrell PH, Pickeral OK, Shue C, Vosshall LB, Zhang J, Zhao Q, Zheng XH, Zhong F, Zhong W, Gibbs R, Venter JC, Adams MC and Lewis S (2000) Comparative genomics of the eukaryotes. Science 287: 2204-2215

13. Kumar S (1999) Mechanisms mediating caspase activation in apoptosis. Cell Death Differ. 6: 1060-1066

14. Kumar S and Colussi PA (1999) Prodomains-adaptors-oligomerization: the pursuit of caspase activation in apoptosis. Trends Biochem. Sci. 24: 1-4

15. Aravind L, Dixit VM and Koonin EV (1999) The domains of death: evolution of apoptosis machinery. Trends Biochem. Sci. 24: 47-53

16. LiP, Nijhawan D, Budihardjo I, Srinivasula SM, Ahmad M, Alnemri ES and Wang $X$ (1997) Cytochrome $c$ and dATP-dependent formation of Apaf-1/caspase- 9 complex initiates an apoptotic protease cascade. Cell 91: 479-489

17. Zou H, Li Y, Liu X and Wang X (1999) An APAF-1-cytochrome c multimeric complex is a functional apoptosome that activates procaspase-9. J. Biol. Chem. 274: $11549-11556$

18. Abrams JM, White K, Fessler LI and Steller H (1993) Programmed cell death during Drosophila embryogenesis. Development 117: 29-43

19. McCall K and Steller H (1998) Requirement for DCP-1 caspase during Drosophila oogenesis. Science 279: 230-234

20. Song Z, Guan B, Bergman A, Nicholson DW, Thornberry NA, Peterson EP and Steller H (2000) Biochemical and genetic interactions between Drosophila caspases and the proapoptotic genes rpr, hid, and grim. Mol. Cell. Biol. 20: 2907-2914

21. Elrod-Erickson M, Misra S and Schneider D (2000) Interaction between the cellular and humoral immune response in Drosophila. Curr. Biol. 10: 781-784

22. Fraser AG, McCarthy NJ and Evan GI (1997) drICE is an essential caspase required for apoptotic activity in Drosophila cells. EMBO J. 16: 6192-6199

23. Meier P, Silke J, Leevers SJ and Evan GI (2000) The Drosophila caspase DRONC is regulated by DIAPI. EMBO J. 19: 598-611

24. Hawkins CJ, Yoo SJ, Peterson EP, Wang SL, Vernooy SY and Hay BA (2000) The Drosophila caspase DRONC is a glutamate/aspartate protease whose activity is regulated by DIAP1, HID and GRIM. J. Biol. Chem. 275: 27084-27093

25. Kanuka H, Sawamoto K, Inohara N, Matsuno K, Okano H and Miura M (1999) Control of the cell death pathway by Dapaf-1, a Drosophila Apaf-1/CED-4-related caspase activator. Mol. Cell 4: 757-769

26. Yang X, Chang HY and Baltimore D (1998) Essential role of CED-4 oligomerisation in CED-3 activation and apoptosis. Science. 281: 1355-1357

27. Rodriguez A, Oliver H, Zou H, Chen P, Wang X and Abrams JM (1999) Dark is a Drosophila homologue of Apaf-1/CED-4 and functions in an evolutionarily conserved death pathway. Nat. Cell Biol. 1: 272-279

28. Zhou L, Song Z, Tittel J and Steller H (1999) HAC-1, a Drosophila homolog of APAF-1 CED-4 functions in developmental and radiation-induced apoptosis. Mol. Cell 4: 745-755 
29. Hawkins CJ, Wang SL and Hay BA (1999) A cloning method to identify caspases and their regulators in yeast: Identification of Drosophila IAP1 as an inhibitor of the Drosophila caspase DCP-1. Proc. Natl. Acad. Sci. USA 96: 2885-2890

30. Kaiser WJ, Vucic D and Miller LK (1998) The Drosophila inhibitor of apoptosis DIAP1 suppresses cell death induced by the caspase drICE. FEBS Lett. 440: $243-248$

31. Wang SL, Hawkins CJ, Yoo SJ, Muller HA and Hay BA (1999) The Drosophila caspase inhibitor DIAP1 is essential for cell survival and is negatively regulated by HID. Cell 98: 453-463

32. Goyal L, MacCall K, Agapite J, Hartwieg E and Steller H (2000) Induction of apoptosis by Drosophila reaper, hid and grim through inhibition of IAP function. EMBO J. 19: 589-597

33. Green DR and Reed JC (1998) Mitochondria and apoptosis. Science 281 1309-1312

34. LiK, LiY, Shelton JM, Richardson JA, SpencerE, Chen ZJ, Wang X and Williams RS (2000) Cytochrome c deficiency causes embryonic lethality and attenuates stress-induced apoptosis. Cell 101: 389-399
35. Varkey J, Chen P, Jemmerson R and Abrams JM (1999) Altered cytochrome C display precedes apoptotic cell death in Drosophila. J. Cell. Biol. 144: 701-710

36. Liu X, Zou H, Slaughter C and Wang X (1997) DFF, a heterodimeric protein that functions downstream of caspase-3 to trigger DNA fragmentation during apoptosis. Cell 89: 175-184

37. Enari M, Sakahira H, Yokoyama H, Okawa K, Iwamatsu A and Nagata S (1998) A caspase activated DNase that degrades DNA during apoptosis, and its inhibitor ICAD. Nature 391: 43-50

38. Yokoyama H, Mukae N, Sakahira H, Okawa K, Iwamatsu A and Nagata S (2000) A novel activation mechanism of caspase-activated DNase from Drosophila melanogaster. J. Biol. Chem. 275: 12978-12986

39. LaCountDJ, Hanson SF, Schneider CL and Friesen PD (2000) Caspase inhibitor P35 and inhibitor of apoptosis Op-IAP block in vivo proteolytic activation of an effector caspase at different steps. J. Biol. Chem. 275: 15657-15664 